\title{
Identifying codes of Cartesian product of two cliques of the same size
}

\author{
S. Gravier* J. Moncel ${ }^{\dagger}$ A. Semri ${ }^{\ddagger}$ \\ Submitted: Sep 10, 2007; Accepted: Feb 4, 2008; Published: Feb 11, 2008 \\ Mathematics Subject Classification: 05C99, 94B60, 94C12
}

\begin{abstract}
We determine the minimum cardinality of an identifying code of $K_{n} \square K_{n}$, the Cartesian product of two cliques of same size. Moreover we show that this code is unique, up to row and column permutations, when $n \geq 5$ is odd. If $n \geq 4$ is even, we exhibit two distinct optimal identifying codes.
\end{abstract}

\section{Introduction}

Given two graphs $G_{1}=\left(V_{1}, E_{1}\right)$ and $G_{2}=\left(V_{2}, E_{2}\right)$, the Cartesian product of $G_{1}$ and $G_{2}$, denoted by $G_{1} \square G_{2}$, is the graph on vertex set $V_{1} \times V_{2}$ such that

- $(x, y)\left(x, y^{\prime}\right) \in E\left(G_{1} \square G_{2}\right)$ if and only if $y y^{\prime} \in E\left(G_{2}\right)$,

- $(x, y)\left(x^{\prime}, y\right) \in E\left(G_{1} \square G_{2}\right)$ if and only if $x x^{\prime} \in E\left(G_{1}\right)$,

- and $(x, y)\left(x^{\prime}, y^{\prime}\right) \notin E\left(G_{1} \square G_{2}\right)$ if $x \neq x^{\prime}$ and $y \neq y^{\prime}$.

In coding theory, it is somehow natural to focus on the Cartesian product, since the most studied metrics (respectively the Hamming and the Lee metrics) in $d$-dimensional spaces can be defined as the iterated Cartesian product of, respectively, cliques and cycles. In this way, the (generalized) hypercube and the torus can be seen as Cartesian products of cliques and cycles, respectively.

In this manuscript, we are interested on identifying codes in Cartesian product of graphs.

*CNRS, ERTé Maths à Modeler, Institut Fourier, 100 rue des maths, 38402 Saint Martin d'Hères, France. e-mail: sylvain.gravier@ujf-grenoble.fr

†INPG, ERTé Maths à Modeler, Laboratoire G-SCOP, 46 Av. Félix Viallet, 38000 Grenoble, France. e-mail: julien.moncel@g-scop.inpg.fr

${ }_{\ddagger}^{\ddagger}$ USTHB, Laboratoire LAID3, OR dept Maths Faculty, BP 32, 16111 El Alia, Algiers (Algeria). e-mail: ahmedsemri@yahoo.fr 
Given a graph $G=(V, E)$, let us denote by $N(x)$ the neighbourhood of $x \in V$, that is, the set of vertices adjacent to $x$. The closed neighbourhood $N[x]$ of $x \in V$ is the union of $x$ and $N(x)$. A subset $C$ of $V$ is said to be an identifying code of $G$ if the sets $N[x] \cap C$ are non-empty and distinct for all $x \in V$.

The notion of identifying code was introduced by Karpovsky, Chakrabarty and Levitin [6] to model a fault-detection problem in multiprocessor systems. For another application to sensor networks consult [9]. The challenging problem is, given a graph $G$, to find a minimum size identifying code of $G$. Identifying codes are closely related to other types of codes, like covering codes (which can be used to construct identifying codes in Hamming spaces, see e.g. $[2,3,6])$. In [7], the authors propose a construction of codes identifying sets of vertices in Cartesian products of graphs. There is a large and fast-growing bibliography on identifying codes, which can be found on Antoine Lobstein's webpage [10].

Even for special structures, determining the minimum cardinality of an identifying code is still an open problem. For instance, only recently, it was proven that the minimum density of an identifying code of the two dimensional grid graph (which can be see as the Cartesian product of two infinite paths) is equal to $\frac{7}{20}[4,1]$. Nevertheless, only partial results are known in the finite case [5].

Additionally, Blass, Honkala and Litsyn proposed in [3] the following natural conjecture : the cardinality of a smallest identifying code in the hypercube will increase with the dimension. This conjecture was only partially solved in [8].

In this note, we determine the size of a minimum identifying code of the Cartesian product of two cliques of the same size. Moreover we show that, up to row and column permutations, there exists a unique minimum identifying code in the case where the size of the cliques is odd.

Theorem 1 Let $C$ be a minimum identifying code of $K_{n} \square K_{n}$. Then $|C|=\left\lfloor\frac{3 n}{2}\right\rfloor$. Moreover, if $n \geq 5$ is odd there is a unique (up to row and column permutations) identifying code with cardinality $\left\lfloor\frac{3 n}{2}\right\rfloor$.

\section{Proof of Theorem 1}

We will note $\{1, \ldots, n\}$ the vertex set of the complete graph $K_{n}$ on $n$ vertices.

First we exhibit identifying codes of cardinality $\left\lfloor\frac{3 n}{2}\right\rfloor$ (see Figure 1 and Figure 2).

Let $D=\{(x, x) \mid x=1, \ldots, n\}$. If $n$ is odd let $A=\left\{(n-x+1, x) \mid x=1, \ldots, \frac{n-1}{2}\right\}$. Otherwise, let $A=\left\{(n-x+1, x) \mid x=1, \ldots, \frac{n}{2}\right\}$.

We will prove that $D \cup A$ is an identifying code of $K_{n} \square K_{n}$. First observe that $D$ is a dominating set since it contains an element of each row. Now we check that each pair of vertices $(x, y),(a, b) \in V\left(K_{n} \square K_{n}\right)$ is separated.

If $a \neq x$ and $a \neq y$ (respectively $b \neq x$ and $b \neq y$ ) then $(a, a) \in N((a, b)) \backslash N((x, y))$ (resp. $(b, b) \in N((a, b)) \backslash N((x, y)))$.

So, since $(a, b) \neq(x, y)$, without loss of generality, we have either $a=b=x$ or $(a=y$ and $b=x($ with $x \neq y))$. 


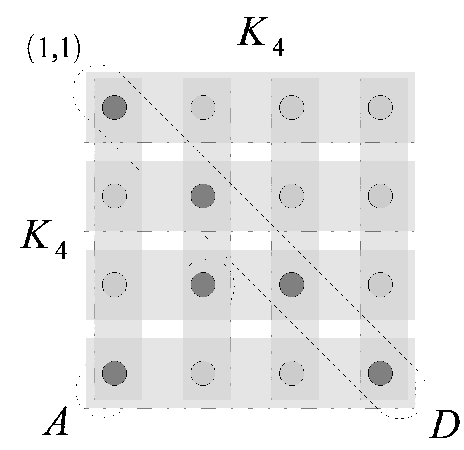

Figure 1: The code $D \cup A$ in the case $n$ even.

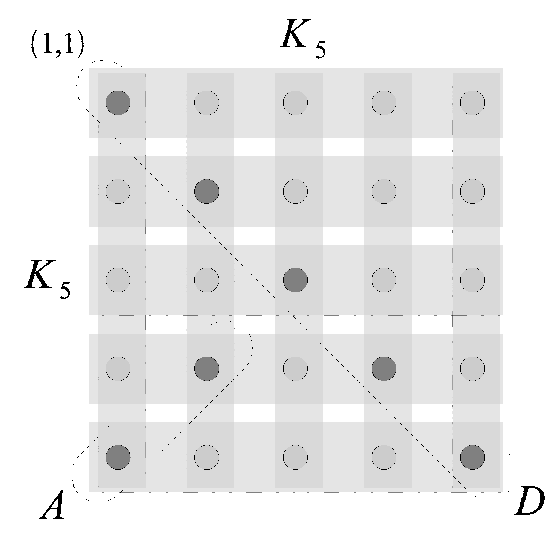

Figure 2: The code $D \cup A$ in the case $n$ odd.

If $a=b=x$ then $(y, y) \in N((x, y)) \backslash N((a, b))$.

Assume, now that $(a, b)=(y, x)$ and $x<y$. First, when $1 \leq x \leq\left\lfloor\frac{n}{2}\right\rfloor,(n+1-x, x) \in$ $N((y, x)) \backslash N((x, y))$. Finally, when $x \geq\left\lceil\frac{n}{2}\right\rceil$ then $(y, n+1-y) \in N((y, x)) \backslash N((x, y))$. This proves that $C$ is an identifying codes of cardinality $|D|+|A|=n+\left\lfloor\frac{n}{2}\right\rfloor=\left\lfloor\frac{3 n}{2}\right\rfloor$.

Let $C$ be an identifying code of $K_{n} \square K_{n}$. We will prove that $|C| \geq\left\lfloor\frac{3 n}{2}\right\rfloor$. Additionally, we show that if $n \geq 5$ is odd and $C$ is a minimum size identifying code then, up to row and column permutations, $C=D \cup A$.

We will need some additional definitions. A row $R_{x}$ for some $x \in\{1, \ldots, n\}$ (respectively column $C_{x}$ ) of $K_{n} \square K_{n}$ is the vertex set $\{(x, i)$ for $i=1, \ldots, n\}$ (resp. $\{(i, x)$ for $i=$ $1, \ldots, n\})$. Let $I(x, y)=N[(x, y)] \cap C$. Remark that if $C$ is an identifying code then $I(x, y) \neq I(u, v)$ for all pairs $(x, y) \neq(u, v)$. A vertex $(x, y)$ which is in $C$ is an $[a, b]$ vertex if $\left|R_{x} \cap C\right|=a$ and $\left|C_{y} \cap C\right|=b$.

We need a simple and useful Lemma :

Lemma 1 There are no two $[*, 1]$-vertices in the same row. By symmetry, there are no two $[1, *]$-vertices in the same column. 
Proof: Suppose that the vertices $(1, x)$ and $(1, y)(x \neq y)$ are $[*, 1]$-vertices, that is to say $(1, x)$ is a $[a, 1]$-vertex, and $(1, y)$ is a $\left[a^{\prime}, 1\right]$-vertex. Then $I(1, x)=I(1, y)$, a contradiction.

As a direct consequence of Lemma 1, one can observe that if there is a row containing exactly one vertex $(x, y)$ of $C$ then the row of any other vertex of $C_{y} \cap C$ contains at least two vertices of $C$.

If $n=1,2$ then trivially the result holds. For $n=3$, it is easy to see that $|C| \geq 4$. It is worth to note that there is another identifying code of cardinality 4 than $D \cup A$ (see Figure 3).

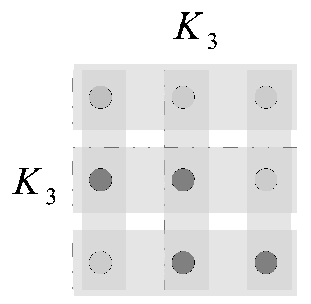

Figure 3: Another optimal identifying code for $n=3$.

So assume now that $n \geq 4$.

Case 1 : there exists a row $R_{x}$ such that $R_{x} \cap C=\emptyset$.

Without loss of generality, we may suppose that $x=1$. If there exists some $u \neq 1$ such that $R_{u} \cap C=\emptyset$ then $I(1, v)=I(u, v)$ for all $v$, which contradicts that $C$ is a identifying code. If there is a $[1, b]$-vertex $(u, v)$ then $I(1, v)=I(u, v)$, a contradiction. Therefore, each row $R_{x}$ with $x \neq 1$ contains at least 2 vertices of $C$, thus $|C| \geq 2(n-1) \geq \frac{3 n}{2}$ if $n \geq 4$.

Moreover, observe that if $n \geq 4$ is odd and $|C|=\left\lfloor\frac{3 n}{2}\right\rfloor$ then $\left|R_{x} \cap C\right| \geq 1$ for all $x$.

Now, by symmetry, one may assume that there is no column $C_{y}$ with $C_{y} \cap C=\emptyset$. Let us now introduce some notations. A 1-row (respectively 1-column) is a row $R_{x}$ (resp. column $C_{y}$ ) such that $\left|R_{x} \cap C\right|=1$ (resp. $\left|C_{y} \cap C\right|=1$ ). A non 1-row (resp. column) will be denoted by $2^{+}$-row (resp. $2^{+}$-column).

Case $2:$ There is no $[1,1]$-vertex.

Let $n_{1}$ be the number of 1-rows and 1-columns and $n_{2}=2 n-n_{1}$.

We claim that $n_{1} \leq n_{2}$. Indeed, associate to each 1-row $R_{x}$ the column $C_{y}$ where $(x, y)=$ $R_{x} \cap C$. Since there is no $[1,1]$-vertex, $C_{y}$ is a $2^{+}$-column. By Lemma 1 , a column $C_{y}$ can not be associated to two distinct 1-rows.

Similarly, one can construct an injection from the set of 1 -columns to the set of $2^{+}$-rows. Thus $n_{1} \leq n_{2}$. Since $n_{1}+n_{2}=2 n$, this implies that:

$$
n_{2} \geq n
$$


Moreover, by double counting, we have:

$$
|C| \geq \frac{n_{1}+2 n_{2}}{2}
$$

By (1) and (2), we obtain that

$$
|C| \geq \frac{2 n+n_{2}}{2} \geq \frac{3 n}{2}
$$

Now, from (3), if $n$ is odd then $|C|>\left\lfloor\frac{3 n}{2}\right\rfloor$ and we are done, which concludes Case 2 .

In order to get the uniqueness result we need the following lemma :

Lemma 2 Let $n$ be an even integer and $C$ be an identifying code of $K_{n} \square K_{n}$. If there is no $[1,1]$-vertex and $|C|=\frac{3 n}{2}$ then, up to row and column permutations, $C=D \cup A$.

Proof: By (1)-(3), we have that $n_{1}=n_{2}=n$ and each $2^{+}$-row (resp. column) contains exactly 2 elements of $C$. Let $r_{1}$ (resp. $c_{1}$ ) be the number of 1-rows (resp. 1-columns). Since $r_{1}+2\left(n-r_{1}\right)=c_{1}+2\left(n-c_{1}\right)=|C|$, then $r_{1}=c_{1}$. Since $r_{1}+c_{1}=n_{1}=n$ then $r_{1}=c_{1}=\frac{n}{2}$. Up to row (resp. column) permutations, one may assume that $R_{1}, \ldots, R_{\frac{n}{2}}$ (resp. $C_{\frac{n}{2}+1}, \ldots, C_{n}$ ) are 1-rows (resp. 1-columns).

Since there is no $[1,1]$-vertex, then for every $x \in\left\{1, \ldots, \frac{n}{2}\right\}$ and $y \in\left\{\frac{n}{2}+1, \ldots, n\right\}$ we have

$$
(x, y) \notin C \quad(A) .
$$

This implies that for every $y \in\left\{\frac{n}{2}+1, \ldots, n\right\}$, we have

$$
\left|\left(C_{y} \cap\left(\cup_{x \in\left\{\frac{n}{2}+1, \ldots, n\right\}} R_{x}\right)\right) \cap C\right|=1 \quad(*) .
$$

Now, by $(A)$ and Lemma 1 , for $x \in\left\{\frac{n}{2}+1, \ldots, n\right\}$, there is at most one $y \in\left\{\frac{n}{2}+1, \ldots, n\right\}$ such that $(x, y) \in C$. By $(*)$, for every $x \in\left\{\frac{n}{2}+1, \ldots, n\right\}$, we have

$$
\left|\left(R_{x} \cap\left(\cup_{y \in\left\{\frac{n}{2}+1, \ldots, n\right\}} C_{y}\right)\right) \cap C\right|=1 \quad(* *) .
$$

Thus, by $(*)$ and $(* *)$, up to column permutations one may assume that $(x, x)$ belongs to $C$ for every $x \in\left\{\frac{n}{2}+1, \ldots, n\right\}$.

Since each row $R_{x}$ with $x \leq \frac{n}{2}$ is a 1-row, then, by Lemma 1, each column $C_{y}$ with $y \leq \frac{n}{2}$ contains at most one element in $C \cap\left\{1, \ldots, \frac{n}{2}\right\} \times\{y\}$. Moreover, by $(A)$, each element $(x, y) \in C$ with $x \in\left\{1, \ldots, \frac{n}{2}\right\}$ satisfies that $y \in\left\{1, \ldots, \frac{n}{2}\right\}$. Here, again, up to row permutations, one may assume that $(x, x) \in C$ with $x \in\left\{1, \ldots, \frac{n}{2}\right\}$.

Now, since each row $R_{x}$ (resp. column $C_{y}$ ) with $x>\frac{n}{2}$ (resp. $y \leq \frac{n}{2}$ ) contains two elements of $C$, up to row (or column) permutations one may assume that $(n-x+1, x) \in C$ for all $x \leq \frac{n}{2}$. 
Now to conclude that, up to rows and columns permutations, $C=D \cup A$ it is enough to first make, say, row permutations on $\left\{R_{\frac{n}{2}+1}, \ldots, R_{n}\right\}$ in order to get $A$.

Case 3 : There exists a $[1,1]$-vertex.

Without loss of generality, suppose that $(1,1)$ is a $[1,1]$-vertex. Observe that $C \backslash\{(1,1)\}$ is an identifying code of the subgraph $G_{2, n}$ induced by $\{2, \ldots, n\} \times\{2, \ldots, n\}$.

If there is a $[1,1]$-vertex $(x, y)$ in $G_{2, n}$ then $I(x, 1)=I(1, y)=\{(1,1),(x, y)\}$, a contradiction.

If $n$ is odd then by Case $2,|C| \geq 1+\frac{3(n-1)}{2}=\left\lfloor\frac{3 n}{2}\right\rfloor$. This shows the first part of Theorem 1. For the uniqueness when $|C|=\left\lfloor\frac{3 n}{2}\right\rfloor$, we apply Lemma 2 as shown in Figure 4.

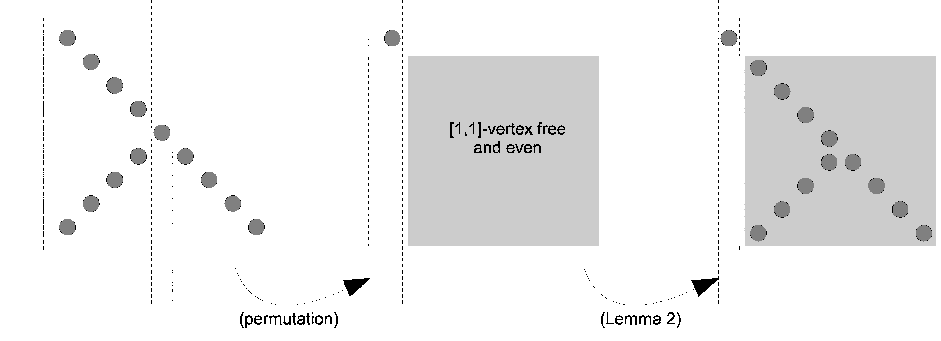

Figure 4: Uniqueness in the case where $n$ is odd.

If $n$ is even then, again, by Case $2,|C| \geq\left\lceil 1+\frac{3(n-1)}{2}\right\rceil \geq\left\lfloor\frac{3 n}{2}\right\rfloor$. This terminates the proof of Theorem 1.

Note that for the case where $n$ is even, there are at least two distinct minimum identifying codes of $K_{n} \square K_{n}$. We exhibited one in Figure 1, in which there is no [1,1]vertex. It is easy to construct another one, having a $[1,1]$-vertex, say $(1,1)$. Indeed, consider the (unique) optimal identifying code $C$ of $G_{2, n}$, and let $(x, x)$ be the unique $[1,1]$-vertex of $C$. It is easy to see that $C \cup\{(1,1),(x, y)\}$ is a minimum identifying code of $K_{n} \square K_{n}$, where $y$ is any coordinate different from $x$.

\section{References}

[1] Y. Ben-Haim, S. Litsyn. Exact minimum density of codes identifying vertices in the square grid, SIAM J. on Discrete Mathematics 19 (2005), 69-82.

[2] U. Blass, I. Honkala, S. Litsyn. On Binary Codes for Identification, J. of Combinatorial Designs 8 (2000), 151-156.

[3] U. Blass, I. Honkala, S. Litsyn. Bounds on Identifying Codes, Discrete Mathematics 241 (2001), 119-128.

[4] G. Cohen, S. Gravier, I. Honkala, A. Lobstein, M. Mollard, C. Payan, G. Zémor. Improved identifying codes for the grids, Electronic J. of Combinatorics 6 (1) (1999) (http://www.combinatorics.org/Volume_6/Html/v6i1r19.html). 
[5] M. Daniel, S. Gravier, J. Moncel, Identifying Codes in Some Subgraphs of the Square Lattice, Theoretical Computer Science 319(1-3) (2004), 411-421.

[6] M.G. Karpovsky, K. Chakrabarty, L.B. Levitin. On a New Class of Codes for Identifying Vertices in Graphs, IEEE Transactions on Information Theory 44(2) (1998), 599-611.

[7] T. Laihonen, J. Moncel. On graphs admitting codes identifying sets of vertices, To appear in Australasian J. of Combinatorics.

[8] J. Moncel. Monotonicity of the Minimum Cardinality of an Identifying Code in the Hypercube, Discrete Appl. Math. 154(6) (2006), 898-899.

[9] S. Ray, D. Starobinski, A. Trachtenberg, R. Ungrangsi. Robust Location Detection with Sensor Networks, IEEE J. on Selected Areas in Communications 22(6) (2004), 1016-1025.

[10] http://www.infres.enst.fr/ lobstein/debutBIBidetlocdom.ps 